\title{
NONLINEAR DEVELOPMENT OF THE KINK INSTABILITY IN CORONAL FLUX TUBES
}

\author{
I. J. D. Craig AND A. D. SNeyd \\ University of Waikato, Hamilton, New Zealand \\ Received 1989 May 31; accepted 1989 December 4
}

\begin{abstract}
Solar prominences and flares are believed to be caused by rapid release of magnetic energy stored in coronal magnetic fields. Recent studies of the linear phase of ideal MHD instabilities has shown that energy release is slow and weak, so it is therefore important to study the nonlinear phase to see if this provides a mechanism for significant energy release. In this paper we describe a Lagrangian numerical scheme for simulating nonlinear evolution of ideal MHD equilibria and apply it to an unstable finite Gold-Hoyle flux tube, line-tied to perfectly conducting endplates. The ensuing kink instability develops considerably faster than linear theory would predict, and eventually (over typically 100 Alfvén time scales) a new kinked equilibrium is attained, in which current sheets appear to be present. Little magnetic energy is lost in the ideal MHD phase, but resistive instabilities in the current sheets could lead to much more explosive energy release. Numerical studies of nonlinear interactions indicate that growth of the unstable kink mode is suppressed by the presence of other modes, which offers a possible explanation of the observed longevity of coronal loops.
\end{abstract}

Subject headings: hydromagnetics - instabilities - Sun: corona - Sun: flares

\section{INTRODUCTION}

A common supposition in astrophysics is that solar flares can be precipitated by the rapid, ideal MHD collapse of coronal magnetic fields. This view is reinforced by the known instability of long, twisted cylindrical flux tubes to helical-kink perturbations (Anzer 1968; Raadu 1972; Hood and Priest 1981). However, the question of the severity of the instabilityin particular the rate and magnitude of the energy release- has only recently been addressed (Craig, McClymont, and Sneyd 1988; Sneyd and Craig 1988; Velli, Einaudi, and Hood 1990). It appears the helical kink is a rather delicate instability, growing and releasing energy quite slowly, at least during the linear phase (Foote and Craig 1990). In particular, for cylindrically symmetric force-free fields in which the twist $T=$ $B_{\theta} / r B_{z}$ is an increasing function of $r$, the rate of magnetic energy release over the linear phase is at least three orders of magnitude smaller than one might anticipate from dimensional estimates. Since a solar flare is a violent event these findings throw into sharp focus the key question of whether finite amplitude effects accelerate or quash the linear instability.

The purpose of the present paper to explore the nonlinear development of the helical kink disturbance. As motivation we note that finite amplitude stabilization of the field could arrest the delicate linear instability at a very low level. This saturation could explain the remarkable longevity of observed coronal loops, but then what is the mechanism for solar flare? Either way our study addresses directly the question of whether coronal loops collapse violently on the MHD time scale of the plasma or whether they evolve to some neighboring stable equilibrium.

The mathematical and computational techniques for determining the nonlinear evolution of three-dimensional coronal magnetic fields have already been developed. Here we employ the Lagrangian scheme of Craig and Sneyd (1986) to quantify the rate and severity of the magnetic energy release during nonlinear development. As initial condition we impose an unstable helical kink displacement for the line-tied Gold-Hoyle flux tube, calculated using the general linear methods of Craig et al. (1988). We emphasize that, although we restrict our attention to a specific equilibrium model, our mathematical and computational techniques are quite general, requiring no prior assumptions as to the symmetry, periodicity, or reduced dimensionality of the problem.

In § II we outline the physical and mathematical basis of the magnetic relaxation scheme. In particular we show that the assumption of "frictional MHD" equations for the relaxation process leads to a parabolic system of equations and point out the physical and computational significance of this result. [The argument for parabolicity given by Craig and Sneyd (1986) contains an error, although the conclusion is correct.] We also outline the computational scheme employed. In $\S$ III the evolution of an unstable Gold-Hoyle flux tube is followed in detail and contrasted with the development of a stable "control" configuration. The ramifications of nonlinear mode interactions are also briefly explored. Finally our conclusions are presented in $\S$ IV.

\section{MAGNETIC RELAXATION METHOD}

\section{a) Introduction}

The concept of magnetic relaxation (Moffatt 1985) is useful for inferring the existence of equilibria, and as a computational strategy for determining the structure and stability of coronal magnetic fields. In this paper we concentrate on the force-free field equations

$$
(\nabla \times B) \times B=0 .
$$

These are nonlinear, and most known analytic solutions involve an ignorable coordinate. The existence of solutions however, in quite general circumstances, can be inferred by a magnetic relaxation thought experiment (Moffatt 1985). Imagine an initial nonequilibrium field in a tenuous perfectly conducting fluid, whose motion involves some form of mechanical dissipation (e.g., viscosity). Under the influence of magnetic forces the fluid will move and the field evolve, the magnetic energy $\boldsymbol{M}$ decreasing monotonically because of 
mechanical dissipation. The magnetic helicity $H$, which is a measure of field line linkages, remains constant during the evolution, and a non-zero value of $H$ implies a positive lower bound on $M$ (Moffatt 1985), so that eventually $M$ must tend to a positive constant, and the field attain a nontrivial $(B \not \equiv 0)$ equilibrium.

However, the final equilibrium may contain current sheets: surfaces across which $\boldsymbol{B}$ is discontinuous. The occurrence of such singularities is of vital physical importance, since they are the source of energetic resistive instabilities and magnetic reconnection. Parker $(1972,1986)$ argues that perturbing even such a simple equilibrium as a uniform field will in general create current sheets, but van Ballegooijen $(1985,1988)$ has pointed out an error in Parker's original argument and claims on the contrary that current sheets cannot occur. Arnol'd (1974) concluded that an equilibrium resulting from magnetic relaxation would not in general be differentiable, and similar conclusions have been reached by Tsinganos, Distler, and Rosner (1984). Recent calculations by Bajer (1989) have shown that current sheets form in a two-dimensional field consisting of four magnetic eddies, and Sneyd (1989) has also described analytic solutions of equation (2.1) in which singularities appear. Thus while the question of whether current sheets must generally be expected remains somewhat contentious, there are many examples of their occurrence.

In a force-free equilibrium, the change in magnetic field direction across a current sheet must be accomplished by means of a continuous (rapid) rotation of the field vector. A simple model for such a sheet is the force-free field

$$
\boldsymbol{B}=[\tanh (k z), \operatorname{sech}(k z), 0]
$$

in the limit as the constant $k \rightarrow \infty$. No neutral points or lines of $B$ can be present. This contrasts with the two-dimensional $X$-type neutral points analyzed by Syrovatski (1971) and the classical Sweet-Parker picture in which a region of high gas pressure straddles the neutral line separating approaching anti-parallel magnetic fields.

The computational implementation of the magnetic relaxation process has been discussed independently by several authors (Chodura and Schlüter 1981; Craig and Sneyd 1986; Yang, Antiochos, and Sturrock 1986). The common feature is that a "frictional" force equation is adopted which guarantees that fluid motions decrease the magnetic energy of the plasma. Thus, in the idealized case of low beta plasma (in which gas pressure effects are negligible), the momentum equation assumes the form

$$
\rho \frac{D v}{D t}=\boldsymbol{J} \times \boldsymbol{B}-\gamma \boldsymbol{v}
$$

where $\gamma$ is a positive coefficient of friction. Without the frictional term the system would oscillate indefinitely with a continual interchange of magnetic and kinetic energies. Since, in a real plasma, dissipation mechanisms always exist that eventually damp MHD oscillations, we can regard the frictional term as mathematical expedient for attaining a physically realizable equilibrium. Bajer (1989) gives a simple example to show that if more than one equilibrium is attainable, then initial conditions, and parameters in the equation of motion, may determine the outcome. It is conceivable therefore that the choice of $\rho$ and $\gamma$ may select one out of a number of possible equilibria, but since a coronal loop experiences a wide variety of initial disturbances, it seems likely that any choice will lead to a physically realizable state.
In this paper we are not concerned with inertial effects so we set $\rho=0$, choose the coefficient of friction equal to unity, and adopt equation (2.2) in the form

$$
\boldsymbol{v}=\boldsymbol{J} \times \boldsymbol{B} .
$$

It is readily shown (Chodura and Schlüter 1981; Craig and Sneyd 1986) that neither the equilibrium solution nor its linear stability properties are falsified in any significant way by the adoption of equation (2.3).

\section{b) Mathematical Classification of the Induction Equation}

It is instructive theoretically, as well as important in terms of developing computational strategies, to investigate the mathematical classification of the magnetic relaxation equations. Combining the frictional equation of motion (2.3) with the ideal MHD induction equation gives

$$
\frac{\partial B}{\partial t}=\nabla \times[(J \times B) \times B]
$$

The mathematical form of this field evolution equation-as opposed to its computational implementation - is most conveniently investigated by adopting an Eulerian representation. Introducing the vector potential $\boldsymbol{B}=\boldsymbol{\nabla} \times \boldsymbol{A}$ in equation (2.3) yields

$$
v=\left[\nabla(\nabla \cdot A)-\nabla^{2} A\right] \times B .
$$

Now if we "uncurl" the induction equation (2.4) we obtain an evolution equation for $\boldsymbol{A}$ viz

$$
\frac{\partial A_{i}}{\partial t}=\left(\delta_{i \alpha} B^{2}-B_{i} B_{\alpha}\right)\left(A_{\alpha, \beta \beta}-A_{\beta, \alpha \beta}\right),
$$

where repeated Greek suffixes are summed from 1 to 3 and $f_{, \alpha}$ for example denotes $\partial f / \partial x_{\alpha}$. In this formulation, however, we cannot impose the guage condition $\nabla \cdot \boldsymbol{A}=0$ as assumed by Craig and Sneyd (1986), but their conclusion that equation (2.6) is a parabolic system still holds as we shall now demonstrate.

To analyze the local character of equation (2.6) we take a three-dimensional Fourier transform, setting

$$
A_{i}(\boldsymbol{x})=(2 \pi)^{-3 / 2} \int \exp (i \boldsymbol{k} \cdot \boldsymbol{x}) \hat{A}_{i}(\boldsymbol{k}) d \boldsymbol{k}
$$

and assume that the coefficient matrix $\delta_{i j} B^{2}-B_{i} B_{j}$ (which involves only first derivatives of $A$ ) is constant. The result is

$$
\frac{\partial \hat{A}_{i}}{\partial t}=\left(\delta_{i \alpha} B^{2}-B_{i} B_{\alpha}\right)\left(k_{\alpha} k_{\beta}-k^{2} \delta_{\alpha \beta}\right) \hat{A}_{\beta}=M_{i \beta} \hat{A}_{\beta},
$$

say, and the local character of the equation depends upon the eigenvalues of $M$. These are shown in the Appendix to be $\left\{-B^{2} k^{2}, 0,-B^{2} k^{2} \cos ^{2} \theta\right\}$ where $\theta$ is the angle between $B$ and $\boldsymbol{k}$. Thus $M$ has no positive eigenvalues, and equation (2.6) is parabolic.

This result has two important implications. Physically it confirms that magnetic instabilities must be entirely global in nature since short-wavelength local perturbations in $\boldsymbol{B}$ must eventually diffuse away. Thus ideal MHD instabilities tend to be slower than expected, as the dominant eigenmode must generally evolve from perturbations which are orchestrated over wavelengths long compared to the length scale of the field variation. Second, the local stability of the field implies that (locally) unconditionally stable numerical schemes must be 
employed if severe and often physically unwarranted restrictions on the computational time step are to be avoided.

Finally we mention that although the Eulerian vector potential formulation provides perhaps the simplest mathematical formulation of the frictional system its computational possibilities have not been explored as yet. The Lagrangian relaxation scheme outlined below has more complicated evolution equations, but this is compensated by the fact that the computational grid moves with the fluid and magnetic field lines during the relaxation process, an advantage shared to some extent by the Eulerian Clebsch variable formulation of Yang et al. (1986).

\section{c) Computational Form of the Fictitious System}

In the Lagrangian scheme of Craig and Sneyd (1986) we use $\boldsymbol{x}(X, t)$ to denote the position at time $t$ of a fluid particle initially at $\boldsymbol{X}$. In ideal MHD, the vector $\boldsymbol{B} / \rho$ evolves in the same way as a fluid line element, which leads to the Cauchy solution

$$
B_{i}=\frac{\partial x_{i}}{\partial X_{\alpha}} B_{0 \alpha} \Delta^{-1}, \Delta=\frac{\partial\left(x_{1}, x_{2}, x_{3}\right)}{\partial\left(X_{1}, X_{2}, X_{3}\right)},
$$

where $\boldsymbol{B}_{0}$ is the initial magnetic field. When this solution is combined with the frictional equation (2.6) we obtain a system of the form

$$
\dot{x}_{i}=A_{i \alpha \beta \gamma} x_{\alpha, \beta \gamma}+C_{i},
$$

where the subscript, $\alpha$, for example, denotes differentiation with respect to $X_{\alpha}$. Here the dependent variables $x_{i}$ are functions defined on a static Cartesian mesh $X_{i}$ and determine the displacement of magnetic field lines in the moving fluid. The explicit expressions for $A$ and $C$ are given (correcting a misprint in the original paper) by

$$
\begin{aligned}
\Delta^{2} A_{i j k l}= & \delta_{i j} B_{0 k} B_{0 l}-b_{i} X_{k, j} B_{0 l} \\
& -b_{j} X_{k, i} B_{0 l}+b^{2} X_{l, i} X_{k, j}, \\
\Delta^{2} C_{i}= & x_{i, \alpha} B_{0 \beta} B_{0 \alpha, \beta}-X_{\alpha, i} x_{\beta, \lambda} b_{\beta} B_{0 \lambda, \alpha},
\end{aligned}
$$

where

$$
b_{i}=x_{i, \alpha} B_{0 \alpha}=\Delta B_{i},
$$

and $X_{i, j}$ denotes $\partial X_{i} / \partial x_{j}$ which are the elements of the matrix inverse of $x_{i, j}$. The system is resolved computationally using an alternating direction implicit scheme (Craig and Sneyd 1988, 1990). This has the advantage of maintaining numerical stability independent of the time step, which can then be chosen purely with regard to accuracy of the solution - a great advantage when approaching an equilibrium, since time steps many orders of magnitude beyond the classical explicit limits can be taken. A further useful property of the numerical system is that $\boldsymbol{\nabla} \cdot \boldsymbol{B} \equiv 0$ and magnetic flux (hence helicity) is automatically conserved throughout the evolution.

To apply the Langrangian scheme to the investigation of kink instabilities in force-free fields it is useful to incorporate a refinement not mentioned in Craig and Sneyd (1986). Since magnetic forces act only in directions perpendicular to the field, motion along the field is effectively unconstrained. This motion is physically uninteresting and computationally inconvenient since it gives rise to needless distortions of the Lagrangian mesh. However, rather than include an isotropic plasma pressure into the problem (which disrupts the integrity of the force-free field calculation by introducing arbitrary parameterizations) we invoke a nonisotropic pressure term that acts only in directions along the magnetic field. We assume for convenience an isothermal relation between $p$ and $\rho$, so that

$$
p=k \rho=k \rho_{0} \Delta^{-1} \quad(k=\text { constant }),
$$

where $\rho_{0}$ is the initial (uniform) density. The additional force introduced is

$$
-\frac{\partial p}{\partial x_{\alpha}} B_{\alpha} B_{i}
$$

which leads to an extra term

$$
k \rho_{0} \Delta^{-1} b_{i} B_{0 l} X_{k, j}
$$

on the right-hand side of equation (2.9).

\section{EVOLUTION OF UNSTABLE GOLD-HOYLE FLUX TUBE}

\section{a) Preliminary Comments}

The computational experiment consists of following the magnetic relaxation of a perturbed force-free equilibrium field. In cylindrical polars the initial field is given by

$$
\boldsymbol{B}=\left(0, \frac{r}{1+r^{2}}, \frac{1}{1+r^{2}}\right) .
$$

In this nondimensional formulation the maximal field strength is normalized to unity while the length scale is chosen as the radial distance over which $B_{z}$ decreases by a factor of 2 . The equilibrium field is "contaminated" by a finite amplitude perturbation, and the relaxation is followed within a rectangular region bounded by perfectly conducting rigid walls $|x|$, $|y|=a, z= \pm l$. The fluid and field lines are fixed at the boundaries $|z|=l$ to model the influence of "line tying" by the dense photosphere. Since the field also penetrates the lateral perfectly conducting boundaries we must also take the field lines to be fixed at $|x|,|y|=a$. It is known from linear studies that provided the lateral boundaries are placed far enough away to negate their stabilizing influence [typically $a>O(10)$ ], the equilibrium (3.1) is unstable for tube lengths $2 l>2.5 \pi$ (Hood and Priest 1981; Foote and Craig 1990). In all results that follow we take $a=15$.

In our first set of results, the applied perturbation is the most unstable kink mode, computed by the methods of Craig et al. (1988). We attempt to follow the evolution until a new stable equilibrium is reached, or until current sheets begin to form, at which point the extreme mesh distortion implies that sufficient accuracy cannot be maintained. It is important to stress that although spatial resolution is necessarily limited, we have adopted the same mesh used by Craig et al. (1988), which is known to give qualitatively correct results for linear stability (compare the fully resolved results of Foote and Craig 1990). Moreover, since the present code is Lagrangian, the evolution of the field toward smaller length scales, is accompanied by shearing and compression of the mesh elements, allowing length scales much smaller than the original mesh size to be represented.

Our main results, presented in $\S$ III $a$, focus on the most damaging initial perturbation - the linear eigenfunction with greatest growth rate. In $\S$ III $b$ we contrast the evolution of a stable tube, and in $\S$ III $c$, in order to examine nonlinear mode interactions, we present results for more general forms of initial perturbation. 


\section{b) Field Evolution Diagnostics}

To anticipate our results slightly, our finite amplitude studies confirm that linear instability provides an accurate guide to the global evolution of the field. In other words, fields which are linearly stable relax dynamically back to the initial equilibrium while unstable fields evolve toward more complex magnetic configurations (possibly involving current sheets). To describe these very different evolution paths we use the orthogonal eigenfunctions $\xi_{i}$ of the linear operator $\boldsymbol{F}$, which gives the magnetic force $\boldsymbol{F}(\xi)$ due to a small displacement $\boldsymbol{\xi}$ from equilibrium. The fluid displacement $\xi$ is then expanded in the form

$$
\xi=c_{1} \xi_{1}+c_{2} \xi_{2}+\cdots \text {. }
$$

Here $\xi_{1}$ is the fastest growing mode, used for the initial perturbation. The coefficients $c_{1}$ and

$$
c_{R}=\left(c_{2}^{2}+c_{3}^{3}+\cdots\right)^{1 / 2},
$$

which is a mean square measure of modes generated by nonlinear interaction, were calculated using the formulae

$$
c_{1}=\int \xi \cdot \xi_{1} d V, \quad c_{R}^{2}=\int \xi \cdot \xi d V-c_{1}^{2} .
$$

A normalized version $c_{n}$ of $c_{R}$ is defined by setting

$$
c_{n}=\frac{c_{R}}{\sqrt{c_{1}^{2}+c_{R}^{2}}} .
$$

Another diagnostic used is $J_{\max }$ - the maximum current intensity over all grid points - which is an indicator of current sheet formation. To measure the mesh distortion we also calculate $\theta_{\min }$, the smallest angle between any pair of faces of each mesh box. This is initially $90^{\circ}$ when the mesh is rectangular, but decreases as the mesh becomes sheared. Another measure of mesh distortion used is $\Delta_{\min }$, the minimum value of $\Delta$ - the volume expansion factor. Initially $\Delta_{\min }=1$, but will generally decrease unless the displacement is everywhere incompressible. To monitor relaxation toward stable Gold-Hoyle equilibria we use $\phi_{\max }$, the maximum angle between $\boldsymbol{B}$ and $\boldsymbol{J}$, and also $\xi_{\max }$, the maximum displacement from equilibrium.

\section{c) Linear Eigenfunction Perturbation for Unstable Tube}

The evolution described in this section was initiated by perturbing an unstable Gold-Hoyle equilibrium of length $2 l=8.57>2.5 \pi$. The chosen perturbation was a small multiple of the most unstable eigenfunction $\xi_{1}$. A mesh consisting of 15 points in the $x$-and $y$-directions, and 21 in the $z$-direction, was used, mesh points being concentrated in the region of strong magnetic field (see Figs $2 a-b$ ) via the nonlinear transformation described in Craig et al. (1988). The eigenvalue corresponding to the calculated eigenfunction is $\lambda=2.4 \times 10^{-4}$ $t_{A}^{-2}$, where $t_{A}$ is Alfvén time.

Figure $1 a$ shows a graph of $c_{1}$ and $c_{n}$, against time. In the initial stages, $c_{1}$ increases approximately exponentially, but considerably faster than linear theory would predict, as indicated by the dashed line. Eventually the rate of increase slows, $c_{1}$ tending to a constant value, indicating that the field is approaching a new equilibrium. The graph of $c_{n}$ shows an initial increase, then a gradual decrease, and toward the end of the calculation a further slow increase. The final value of $c_{n}$ shows that the initial $\xi_{1}$ mode still accounts for $\sim 80 \%$ (in a mean square sense) of the final fluid displacement.

The time coordinate in Figure $1 a$ is pseudo time - an artifact of the frictional equation of motion. A comparison between pseudo time $t$ and Alfvén time $t_{A}$ can be made by noting that a mode growing in pseudo time as $\exp (\lambda t)$ would grow in Alfvén time as $\exp \left(\lambda^{1 / 2} t_{A}\right)$. Thus we can write $t_{A}=\lambda^{-1 / 2} t$, and during the rapid development stage of the kink, $\lambda \approx 10^{-3}$ so that $t_{A} \approx 3.16 \times 10^{-2} t$. The total time taken to reach the new equilibrium is therefore $\sim 160$ Alfvén times.

Figure $1 b$ shows a graph of $J_{\max }$ against time. Over the initial stages $J_{\max }$ has an almost constant value of 2.0 , which is the maximum current in the Gold-Hoyle equilibrium. Toward the end of the calculation $J_{\max }$ begins to increase rapidly, finally attaining a value of $\sim 15.0$. The calculation was abandoned at this point since the fluid displacement was practically constant - only $J_{\max }$ was still increasingly rapidly. This implies that $J_{\max }$ is essentially a discontinuous function of the fluid displacement. Such behavior is consistent with current sheet formation in the new equilibrium, and as a further diagnostic

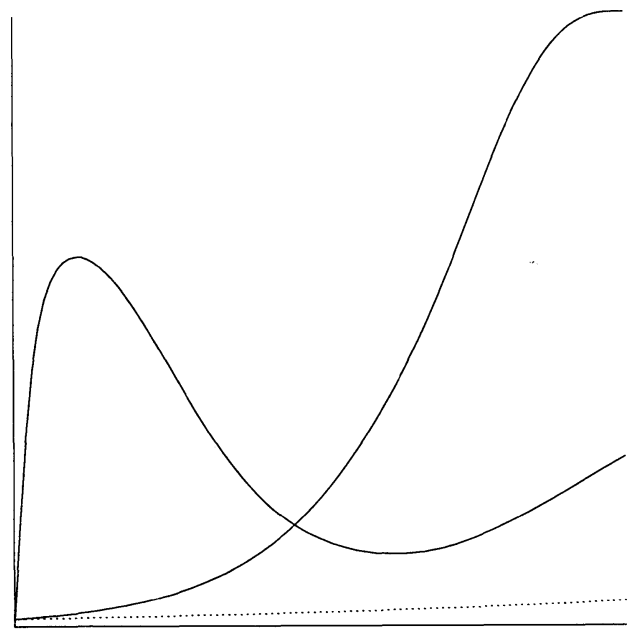

FIG. $1 a$

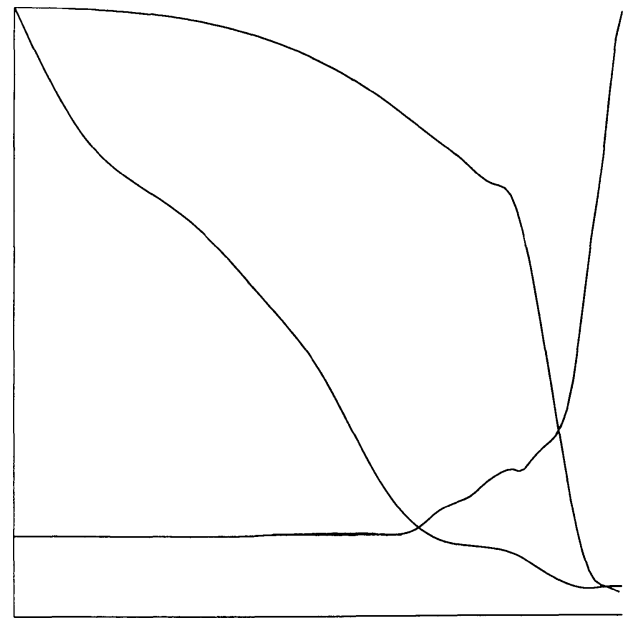

FIG. $1 b$

FIG. 1. - (a) Graphs of $c_{1}$ and $c_{n}$ against $t$ for the unstable tube. The dashed curve shows a graph of $c_{1}$ as predicted by linear theory, namely $c_{1}=0.05 \times$ exp $(\lambda t)$ where $\lambda=2.4 \times 10^{-4}$. (b) Graphs of $J_{\max }$ (the maximum current intensity), $\theta_{\min }$ (the minimum mesh angle) and $\Delta_{\min }$ (the value of $\Delta$ where mesh compression is most intense) against $t$ for the same computation. 


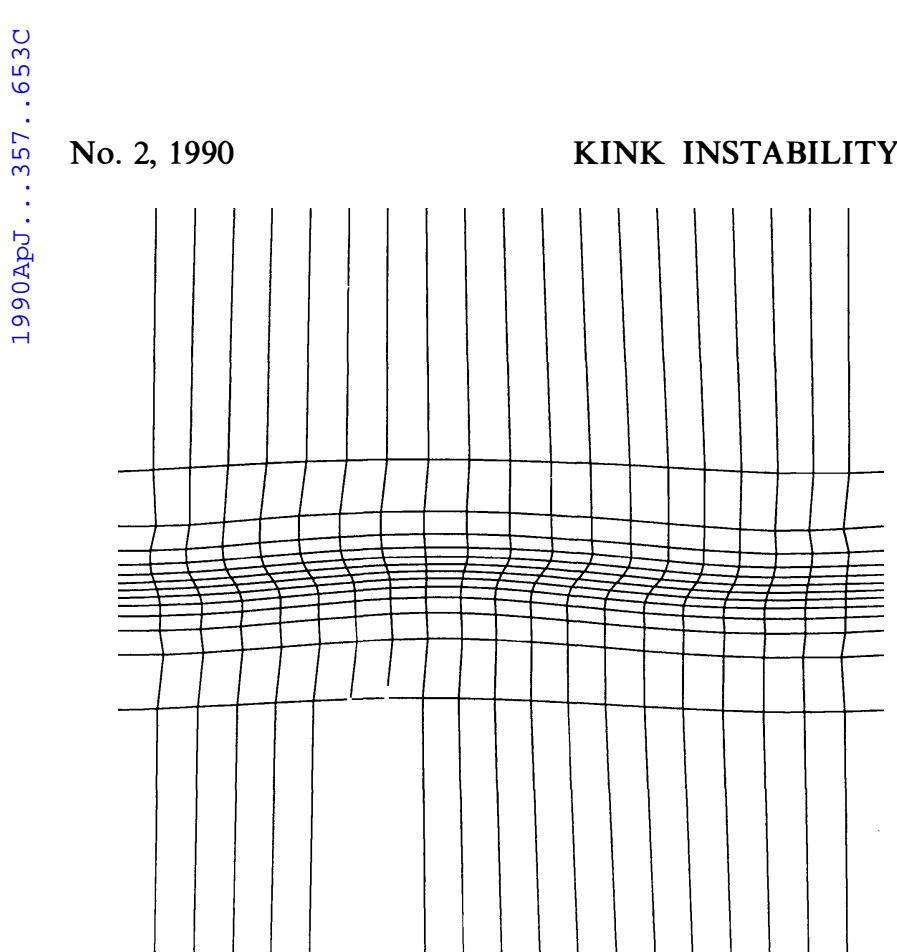

FIG. $2 a$

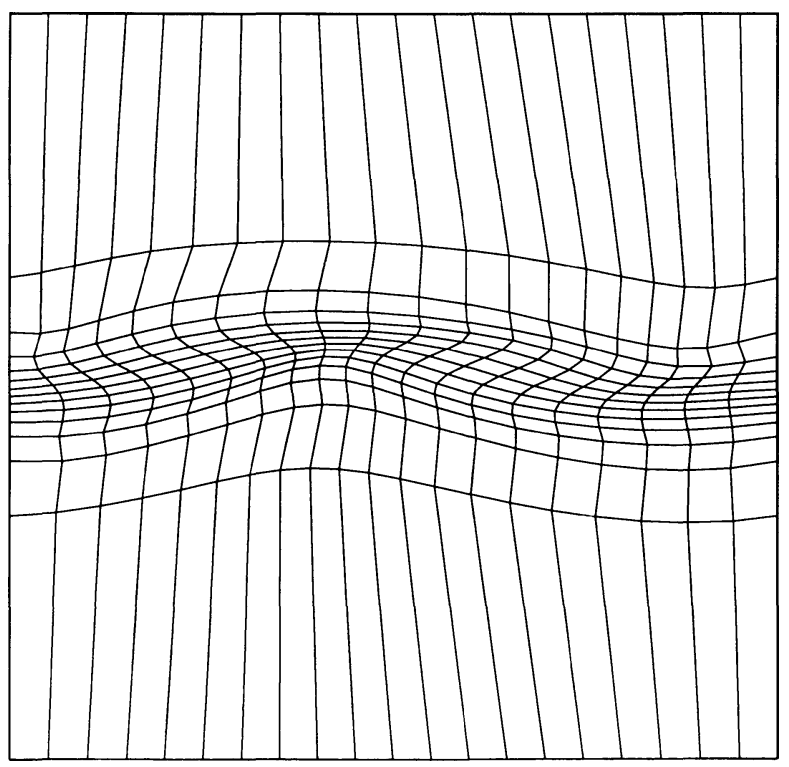

FIG. $2 c$

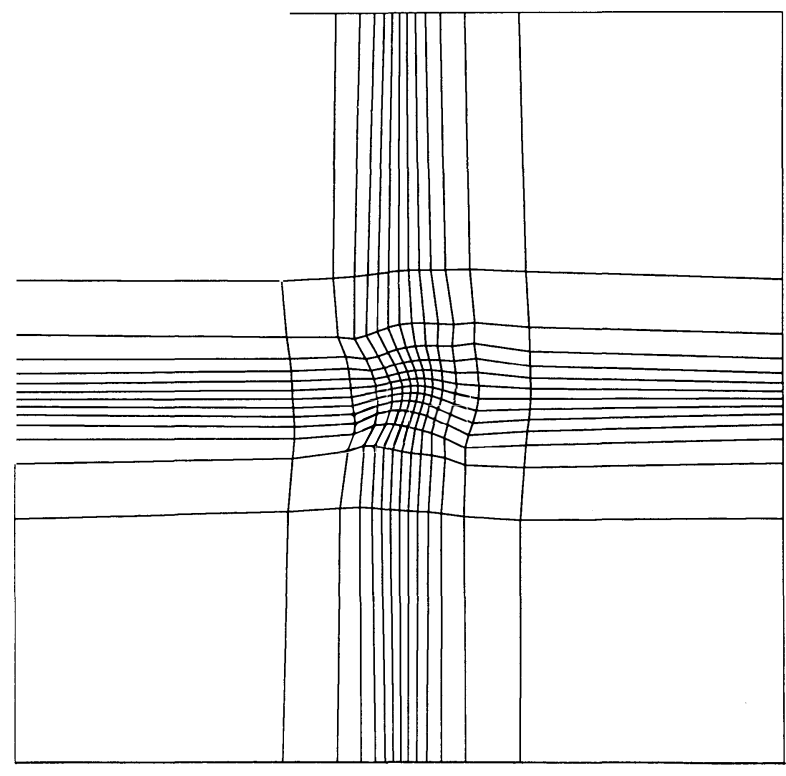

Fig. $2 b$

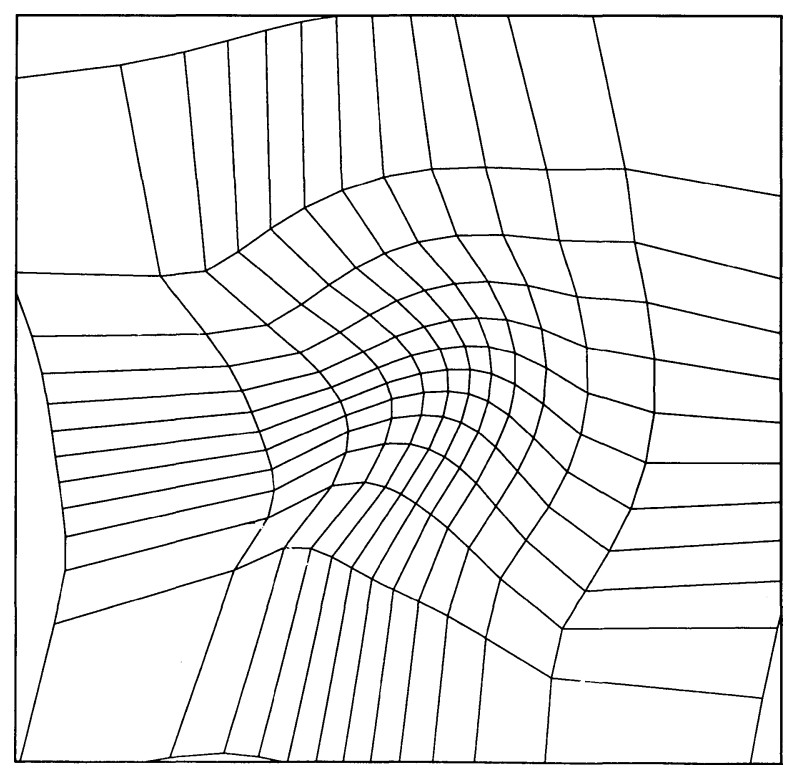

Fig. $2 d$

Fig. 2.- $-(a-g)$ Development of the kink instability for $a=15$ and total tube length $2 l=8.57$. The figures show various plane sections of the computational grid at

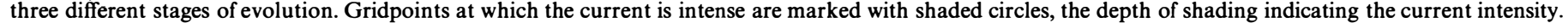
Note that $(d)$ and $(f)$ show only the inner mesh region, $|x|,|y| \leq 8.6$.

$\theta_{\min }$ is also plotted in Figure $1 b$. Initially $\theta_{\min }=90^{\circ}$ but as the final equilibrium is approached, $\theta_{\min }$ tends to a value of $\sim 4^{\circ}$, showing that the mesh boxes are becoming highly sheared. The current sheet is formed as opposite box faces, on which the magnetic field directions are different, are pressed closer and closer together by the shearing of the box. Figure $1 b$ also shows a graph of $\Delta_{\min }$, which is initially 1 but eventually tends to a value of $\sim 5 \times 10^{-2}$, indicating that compression as well as shear is an important factor in generating the current sheet.

To visualize the development of the kink instability, Figure 2 shows longitudinal sections and cross sections of the computational grid at various stages of evolution. The diagrams consist of a network of lines joining gridpoints - i.e. fluid particleslying initially in planes $Y=$ constant or $Z=$ constant. In the course of the kink evolution, the fluid particles cease to be coplanar, so the figures are projections onto a coordinate plane, and any apparent crossing of grid lines is purely a projection effect. In the longitudinal sections ( $Y=$ constant) the horizontal lines are field-line projections. In the later stages of evolution the flux tube becomes severely bent and twisted, and regions of intense electric current are indicated by various degrees of shading around the gridpoints. The current sheets appear to form near each end of the loop (i.e., near the photosphere) where mesh compression and shear is most 


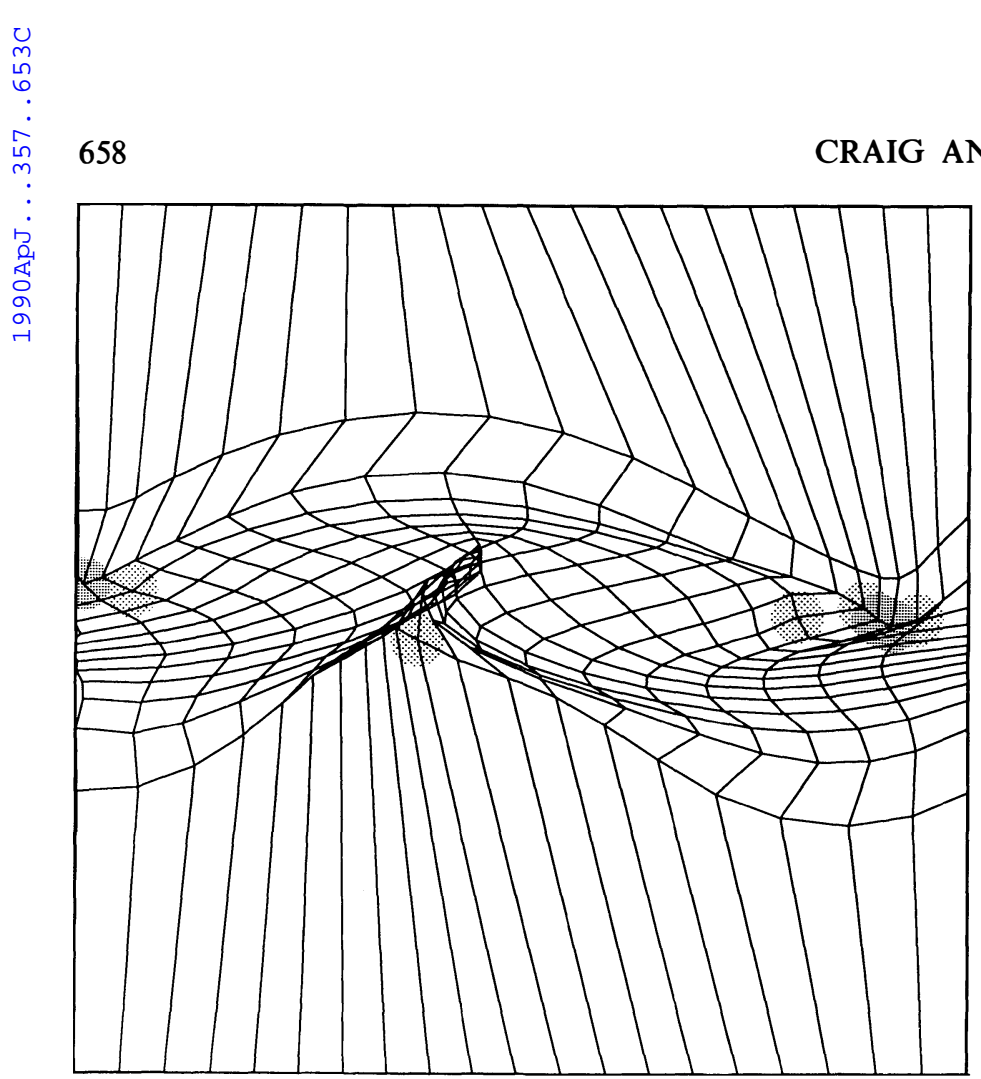

FIG. $2 e$

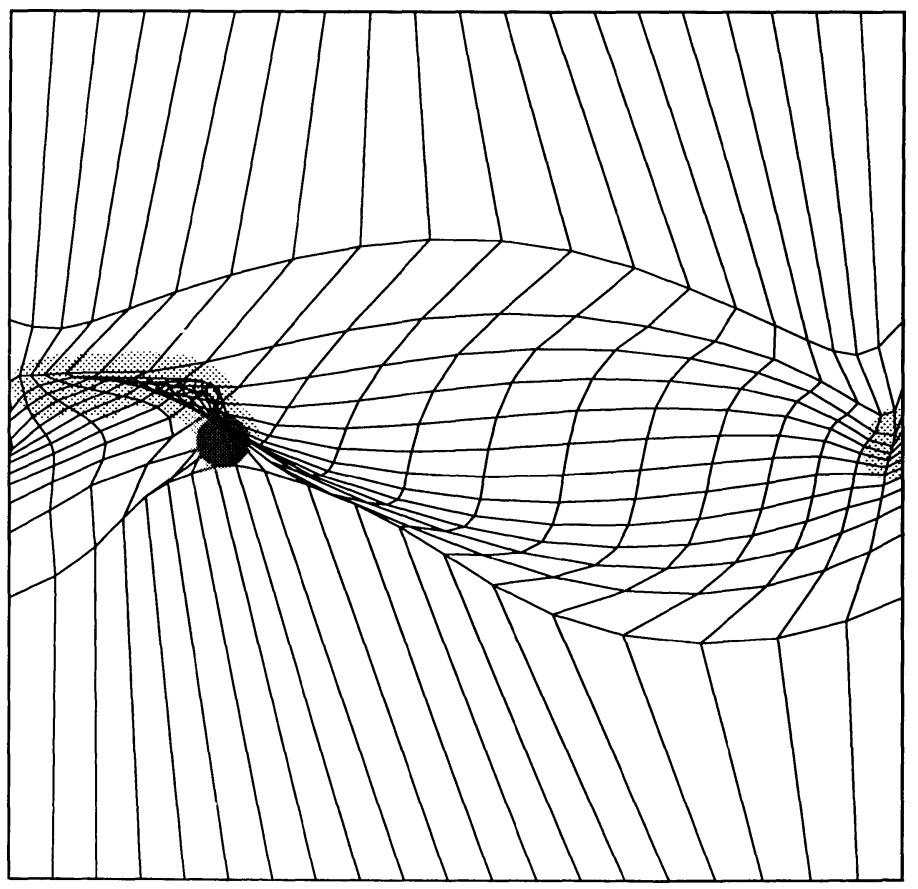

FIG. $2 g$

FIG. 2-Continued

apparent. The additional longitudinal section Figure $2 g$ is shown since this includes the region where electric current is most intense.

The magnetic energy released during magnetic relaxation, was calculated by integrating the power of the Lorentz force $(\boldsymbol{J} \times \boldsymbol{B}) \cdot v$ over space and time. The results proved somewhat unreliable since the magnitude $F$ of the Lorentz force varied considerably with the time step chosen-for small time steps $F$
Vol. 357

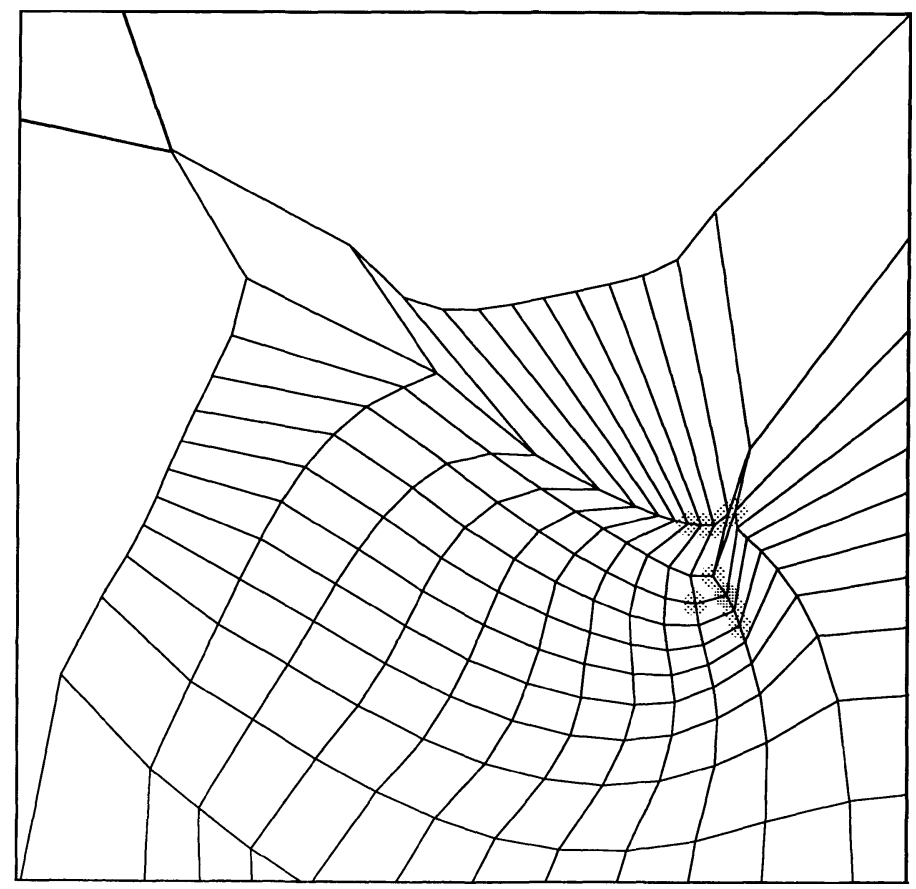

Fig. $2 f$

was always very small, while for larger time steps $F$ could increase by as much as an order of magnitude, indicating that the true evolution was not accurately followed. Apparently the evolution of the kink follows a near-equilibrium path, and small deviations from this path give rise to much larger relative errors in $\boldsymbol{F}$. (Nevertheless the path deviations caused by choice of a large time step did not compromise the overall course of the kink evolution.) Since we generally chose as large a time step as possible to reduce the (considerable) computation time, our results for energy release were overestimates, but it was still clear that the figure was quite small-less than $\sim 5 \%$ of the total magnetic energy. We also experimented with calculating changes in magnetic energy by integrating $B^{2} / 2$ over the mesh, but since the changes were small and the mesh became greatly distorted, the integral was not sufficiently accurate.

\section{d) Perturbations in a Stable Tube}

As a comparison we have performed similar calculations, at identical space resolution, for a stable flux tube. The tube length chosen was $2 l=2.57<2.5 \pi$, with 15 grid points in the $x$ - and $y$-directions, and seven in the $z$-direction. The initial perturbation was a multiple of the most slowly decaying linear normal mode, and was calculated using the methods of Craig et al. (1988) on an identical computational grid.

Figure 3 shows that both $\phi_{\max }$ and $\xi_{\max }$ decay to zero as expected. The decay is rather slow since the most slowly decaying mode is very close to marginal stability, and restoring forces are very weak. Figure 3 also shows a graph of $\phi_{\max }$ against $t$ for the unstable tube of $\S$ III $a$, and the behavior is quite different. The initial large angle $\left(\sim 12^{\circ}\right)$ between $B$ and $J$ decreases rapidly, indicating a settling-down period as the initial perturbation relaxes to near equilibrium; then $\phi_{\max }$ increases gradually as the kink instability gathers momentum. In theory $\phi_{\max }$ should tend to zero again as the new equilibrium is approached, but the onset of current sheets and lack 


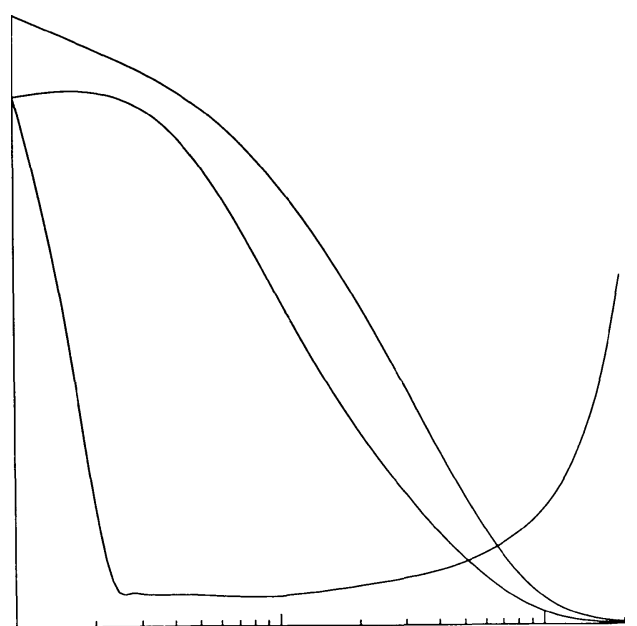

FIG. 3. - Graphs of $\phi_{\max }$ (the maximum angle between $\boldsymbol{B}$ and $\boldsymbol{J}$ ) and $\xi_{\max }$ (the maximum mesh displacement from Gold-Hoyle equilibrium) against $t$ for stable and unstable flux tubes. Note that the time scale is logarithmic.

of resolution means that the angles cannot be calculated with sufficient accuracy.

\section{d) Nonlinear Interactions}

Any physical initial perturbation will consist of a superposition of many eigenmodes, and it is important to understand whether the initial presence of other modes interferes with the development of $\xi_{1}$. We experimented with two initial perturbations described by analytic formulae. The first was a simple " bend," given by the formula

$$
\xi_{a}=\cos \left(\frac{\pi z}{2 l}\right) \cos \left(\frac{\pi x}{2 a}\right) \cos \left(\frac{\pi y}{2 a}\right) \hat{x} .
$$

The second was a helical-kink displacement given by the formula

$$
\xi_{b}=\left[(f(r) \cos \psi \hat{r}-g(r) \sin \psi \hat{\theta}+r g(r) \sin \psi \hat{z}] \cos \left(\frac{\pi z}{2 l}\right),\right.
$$

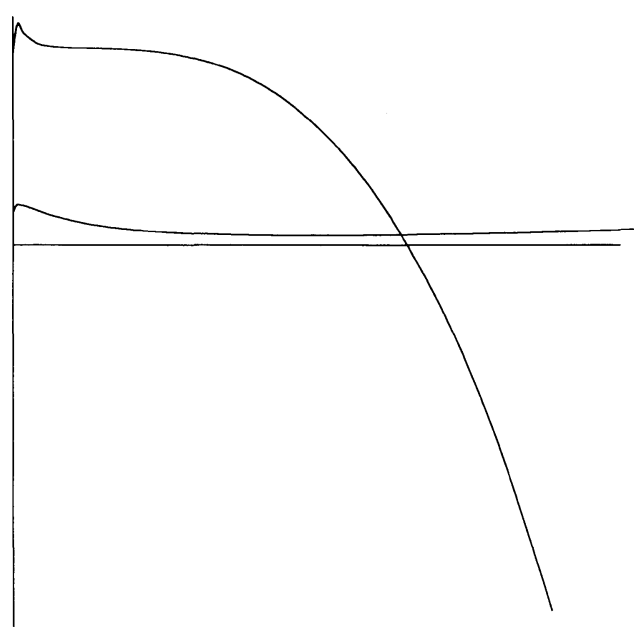

FIG. $4 a$ in cylindrical polar coordinates $(r, \theta, z)$, where

$$
\psi=\theta-k z, f(r)=\begin{gathered}
\left(1-r^{2} / a^{2}\right) \\
\left(1+r^{2}\right)
\end{gathered}, \quad g(r)=\begin{gathered}
f(r)\left(1-r^{2}\right) \\
1+r^{2}
\end{gathered} .
$$

(To ensure that $\xi_{b}=0$ on the boundaries $|x|,|y|=a$ the function $f$ was defined to be zero for $r \geq a$.) The displacement $\xi_{b}$ was designed to mimic the form of the most unstable $m=1$ mode for the infinite Gold-Hoyle flux tube, as described by Sneyd and Craig (1988), but multiplied by the cosine factor to ensure $\xi_{b}=0$ on the endplates $|z|=l$. The wavenumber $k$ was chosen to be 0.5 , as this value seemed to maximize the initial $\xi_{1}$-component $c_{1}$.

Figure $4 a$ shows the growth of the most unstable mode (as measured by the coefficient $c_{1}$ ) for instabilities initiated by multiples of the above displacements. In both cases the maximum initial mesh displacement is 0.2 . With the $\xi_{a}$ displacement, $c_{1}$ increases slightly, decays to zero, and then begins to grow rapidly in the negative sense. In the $\xi_{b}$ case the initial value of $c_{1}$ is smaller (somewhat surprising since this form of disturbance was purposely designed to mimic the most unstable mode); $c_{1}$ again grows briefly, then decreases, but eventually begins to grow slowly again. In both cases the coefficient $c_{n}$ (the mean square measure of displacement in modes $\left.\xi_{2}, \xi_{3} \ldots\right)$ is almost exactly unity, indicating that $\xi_{1}$ comprises a very small proportion of the total displacement. Figure $4 b$ shows a mesh cross-section during the evolution of the $\xi_{a}$ displacement, which indicates that most of the residual displacement is in the outer regions of the flux tube, where the magnetic field and restoring forces are weak.

Note that in Figure $4 a$ the time coordinate is pseudo time. Since nonlinear interactions are important it is not possible to make a simple comparison with Alfvén time. However, we can generally conclude that the presence of other eigenmodes seems to prevent early growth of the most unstable mode.

\section{CONCLUSIONS}

We have used a magnetic relaxation technique to study the nonlinear evolution of three-dimensional ideal MHD insta-

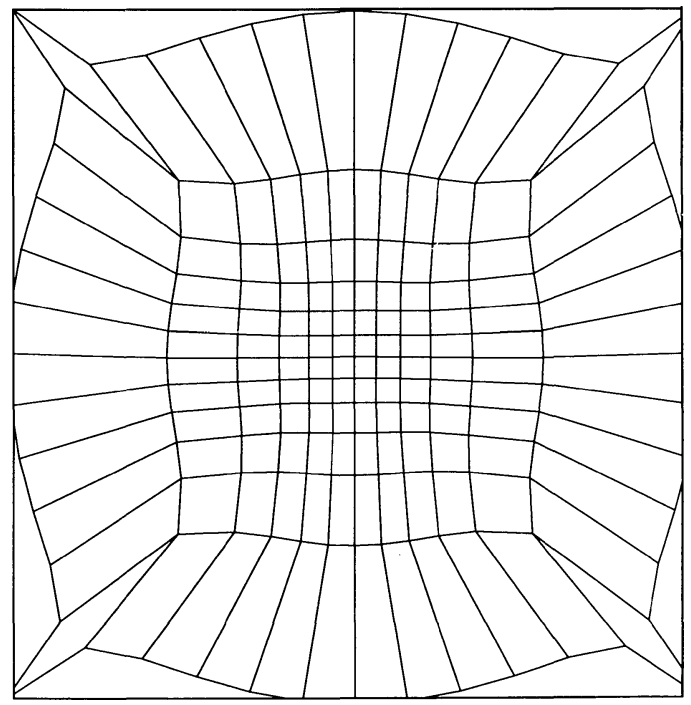

FIG. $4 b$

FIG. 4. - (a) Graphs of $c_{1}$ against $t$ for the initial displacements $\xi_{a}$ and $\xi_{b}$. (b) Mesh section $Z=0$ for $\xi_{a}$ at $t=1300$. 
bilities. Since the relaxation method is Lagrangian, using a grid of fluid particle positions as basic variables, the evolution of small length-scale structures can be represented more accurately than by means of a fixed (Eulerian) mesh. The scheme preserves magnetic flux and therefore magnetic helicity identically. It incorporates an ADI technique to integrate the evolution equations forward in time, so that relatively large time steps may be taken unhindered by numerical stability restrictions; in practice typical time steps may be three orders of magnitude beyond the explicit stability limit.

To gain insight into nonlinear processes in coronal loops, we have applied the Lagrangian relaxation method to the kink instability in an unstable, line-tied, Gold-Hoyle flux tube. Nonlinear forces accelerate the kink quite strongly and are important even in the early stages of development. This could be anticipated since linear theory predicts forces of order $\lambda \xi$, where $\lambda$, the eigenvalue of the most unstable linear mode, $=2.4 \times 10^{-4}$ in our chosen example. The strongest nonlinear forces are of order $\xi^{3}$, and become equally important when $\xi=O\left(10^{-2}\right)$-i.e., at quite modest amplitudes. Eventually the kinked flux tube reaches a new equilibrium which appears to contain current sheets.

Current sheets appear mostly near the tube ends. They are formed by the close approach of magnetic surfaces, partly due to compression, and partly to shearing. It should be emphasized that the maximum compression factor in our calculation is $\sim 20$, while in a coronal loop compression factors of at least $10^{3}$ would be necessary if plasma pressure were to become comparable with magnetic pressure. Moreover, a current sheet can be formed by means of pure shear, even in an incompressible flow, so we would not expect plasma pressure to prevent current sheets.
An important conclusion is that little magnetic energy (somewhat less than $5 \%$ of the total available) is released in the ideal MHD phase of development. However, with the appearance of current sheets, more devastating resistive instabilities will take effect. The role of ideal MHD can therefore be seen as driving the field toward a singular configuration, with length scales small enough to cause magnetic reconnection and violent energy release.

Our studies of nonlinear interactions indicate that the most unstable mode (in linear theory) may be damped in the presence of other modes. Real coronal loops are subject to continual random perturbations, which could keep the kink instability suppressed over long periods, thus explaining the observed longevity of the loops. Another possibility, which follows from the inherent weakness of the kink, is that its nonlinear development could be suppressed by neighboring stable loops in the active region assemblage. However, if isolated loops do exist and these are driven into instability by some mechanism-say by photospheric twisting of the footpoints - then the field will distort and release magnetic energy relatively slowly over, say, at least $10^{2}$ Alfvén times. Either way, since the ideal MHD phase is effective mainly in driving the field toward a configuration involving current sheets, it remains for resistive effects to release the bulk of the stored magnetic energy.

We gratefully acknowledge support from the New Zealand University Grants Committee, and University of Waikato Research Committee, for the purchase of the Microvax II computers used for our computations.

\section{APPENDIX}

\section{EIGENVALUES OF $M$}

We note first that $M$ can be written in the form

$$
M=-B^{2} k^{2} P_{B} P_{k},
$$

where the matrix $P_{B}$ projects any vector onto the plane normal to $B$, and similarly for $\boldsymbol{P}_{k}$. We now consider the eigenvalues of $\boldsymbol{P}_{\boldsymbol{B}} \boldsymbol{P}_{\boldsymbol{k}}$.

Obviously $\boldsymbol{k}$ is an eigenvector with corresponding eigenvalue 0 . Furthermore a vector along the line of intersection of the planes

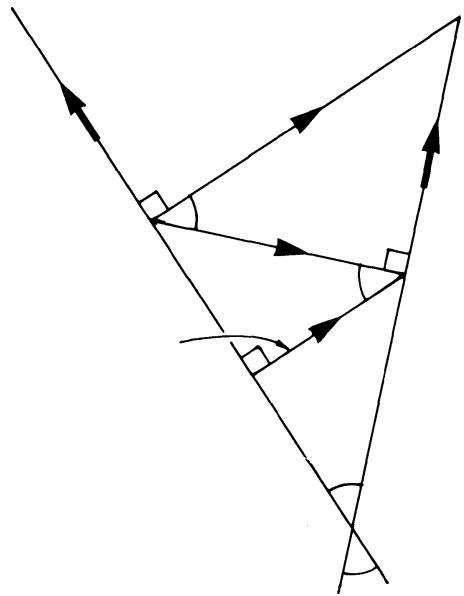

Fig. 5.-Geometrical construction for finding eigenvalue of $P_{B} P_{k}$. 
normal to $\boldsymbol{k}$ and $\boldsymbol{B}$ is invariant under both projection operations and is therefore an eigenvector with corresponding eigenvalue 1. Since $P_{B} P_{k}$ is real and eigenvalues must occur in complex conjugate pairs, it follows that the third eigenvector is also real, say $\lambda$. The general solution for $x$ of $P_{k} x=y$ is $x=y+\alpha k$, where $\alpha$ is any scalar, so the solution of the eigenvalue equation

$$
P_{B} P_{k} x=\lambda x \text { is } x=\lambda x+\alpha k+\beta B .
$$

Thus, either $\lambda=1$ which implies that $B$ and $\boldsymbol{k}$ are parallel, in which case the eigenvalue 1 is repeated, having an eigenspace of dimension 2. Alternatively if $\lambda \neq 1$ then $\boldsymbol{x}$ lies in the plane of $\boldsymbol{B}$ and $\boldsymbol{k}$. It also follows from equation (A2) that $\boldsymbol{x}$ must be perpendicular to $B$, and the geometric construction in Figure 5 shows that $\lambda=\cos ^{2} \theta$, where $\theta$ is the angle between $B$ and $\boldsymbol{k}$.

From equation(A1) the eigenvalues of $M$ are therefore

$$
\left\{-B^{2} k^{2}, 0,-B^{2} k^{2} \cos ^{2} \theta\right\} \text {. }
$$

\section{REFERENCES}

Anzer, U. 1968, Solar Phys., 3, 298.

Arnol'd, V. I. 1974, in Proc. Summer School in Differential Equations (Erevan:

Raadu, M. A. 1972, Solar Phys., 22, 425.

Armenian SSR Academy of Science).

Bajer, K. 1989, Ph.D. thesis, University of Cambridge

Chodura, R., and Schlüter, A. 1981, J. Comput. Phys., 41, 68.

Craig, I. J. D., McClymont, A. N., and Sneyd, A. D. 1988, Ap. J., 335, 441.

Craig, I. J. D., and Sneyd, A. D. 1986, Ap. J., 311, 451.

. 1988, Comput. Math. Appl., 16, No. 4, 341.

1990, Comput. Math. Appl., to appear.

Foote, B. J., and Craig, I. J. D. 1990, Ap. J., 350, 437.

Hood, A. W., and Priest, E. R. 1981, Geophys. Ap. Fluid Dyn., 17, 297.

Parker, E. N. 1972, Ap. J., 174, 499.

1986, Geophys. Ap. Fluid Dyn., 34, 243

Sneyd, A. D. 1989, Geophys. Ap. Fluid Dyn., in press.

Sneyd, A. D., and Craig, I. J. D. 1988, Ap. Space Sci., 151, 265

Syrovatski, S. I. 1971, Soviet Phys.-JETP Letters, 33, 933.

Tsinganos, K. C., Distler, J., and Rosner, R. 1984, Ap. J., 278, 40a.

van Ballegooijen, A. A. 1985, Ap. J., 298, 421.

- 1988, Geophys. Ap. Fluid Dyn., 41, 181.

Velli, M., Einaudi, G., and Hood, A. W. 1990, Ap. J., 350, 428

Yang, W. H., Sturrock, P. A., and Antiochos, S. K. 1986, Ap. J., 309, 383.

I. J. D. Craig and A. D. SNEyd: University of Waikato, Private Bag, Hamilton, New Zealand 